\title{
Editorial
}

\section{Nonlinear Fluid Flow and Heat Transfer}

\author{
O. D. Makinde, ${ }^{1}$ R. J. Moitsheki, ${ }^{2}$ R. N. Jana, ${ }^{3}$ B. H. Bradshaw-Hajek, ${ }^{4}$ and W. A. Khan ${ }^{5}$ \\ ${ }^{1}$ Faculty of Military Science, Stellenbosch University, Private Bag X2, Saldanha 7395, South Africa \\ ${ }^{2}$ School of Computational and Applied Mathematics, University of the Witwatersrand (WITS), Private Bag 3, \\ Johannesburg 2050, South Africa \\ ${ }^{3}$ Department of Applied Mathematics, Vidyasagar University, Midnapore 721 102, India \\ ${ }^{4}$ School of Information Technology and Mathematical Sciences, University of South Australia, Mawson Lake Campus, \\ Mawson Lakes, SA, Australia \\ ${ }^{5}$ Department of Engineering Sciences, Pakistan Navy Engineering College, National University of Sciences \& Technology, \\ Karachi 75350, Pakistan
}

Correspondence should be addressed to O. D. Makinde; makinded@gmail.com

Received 11 June 2014; Accepted 11 June 2014; Published 24 June 2014

Copyright (c) 2014 O. D. Makinde et al. This is an open access article distributed under the Creative Commons Attribution License, which permits unrestricted use, distribution, and reproduction in any medium, provided the original work is properly cited.

In order to stimulate fluid flow, heat transfer, and other related physical phenomena, it is necessary to describe the associated physics in mathematical terms. Nearly all the physical phenomena of interest are obtained by principles of conservation and are expressed in terms of nonlinear partial or ordinary differential equations expressing these principles. For example, the momentum equations express the conservation of linear momentum; the energy equation expresses the conservation of total energy. These nonlinear differential equations which model the dynamics of fluid motion and heat transfer do arise in many areas such as optics, plasma physics, and even traffic flow. Thus, the underlying mathematics has relevance in many branches of science and technology. Generally, the solutions for these complicated nonlinear differential equations can be obtained numerically in most cases; however, analytical solutions for fluid flow and heat transfer problem can still play a very important role in science and engineering, even in the current age of supercomputer. This is because analytical solutions have the big advantage of revealing directly the parameters which influence the solution. This special issue is focused on nonlinear analysis and numerical simulation of typical conservation equations modelling physical phenomena with respect to fluid flow and heat transfer. The original papers explored include a wide variety of topics such as boundary layer flows, nanofluids dynamics, reactive flows, hydromagnetic flows, physiological flows, thermodynamics analysis of fluid flows,
Newtonian and non-Newtonian flows, and nonlinear heat transfer in solids. In "Spectral relaxation method and spectral quasilinearization method for solving unsteady boundary layer flow problems," S. S. Motsa et al. numerically solved model problems of unsteady boundary layer flow caused by an impulsively stretching sheet and the unsteady MHD flow and mass transfer in a porous space using a spectral relaxation method (SRM) and spectral quasilinearisation method (SQLM). It was shown that the SRM is significantly more computationally efficient than the SQLM which, in turn, is faster than the Keller-box method. In "Application of successive linearisation method to squeezing flow with bifurcation," S. S. Motsa et al. employed a computational approach known as successive linearization method (SLM) to tackle a fourth-order nonlinear differential equation modelling the transient flow of an incompressible viscous fluid between two parallel plates produced by a simple wall motion. The solution branches as well as a turning point in the flow field were accurately obtained. Moreover, the study revealed that the proposed SLM approach converges rapidly to the solution of the original nonlinear problem and can be used to solve many other nonlinear equations arising in fluid flow and heat transfer problems. In "Higher order compact finite difference schemes for unsteady boundary layer flow problems," P. G. Dlamini et al. investigated two nonlinear partial differential equations governing the unsteady MHD boundary layer flow and heat transfer over an impulsively stretching surface using 
the compact finite difference relaxation method (CFDRM). It was shown that the CFDRM is computationally faster than the Keller-box method and CFDRM gives highly accurate solutions on coarser grids. Moreover, the results obtained are in good agreement with results obtained using the Kellerbox. In "Classification of the group invariant solutions for contaminant transport in saturated soils under radial uniform water flows," M. M. Potsane and R. J. Moitsheki analysed a nonlinear macroscopic deterministic model describing contaminant transport in saturated soils under uniform radial water flow backgrounds using classical Lie point symmetries. A number of exotic Lie point symmetries are admitted. Group invariant solutions are classified according to the elements of the one-dimensional optimal systems. The symmetry solutions are obtainable when dispersion coefficient is a constant or is given by Taylor's theory of mixing in soils. In "A spectral relaxation approach for unsteady boundarylayer flow and heat transfer of a nanofluid over a permeable stretching/shrinking sheet," S. S. Motsa et al. utilised both the spectral relaxation method and the spectral quasilinearisation method to numerically solve the highly nonlinear equations that describe the unsteady heat transfer in a nanofluid over a permeable stretching or shrinking surface. The results revealed that dual solutions for nanofluid fluid velocity profiles, temperature profiles, and nanoparticles volume fractions exist for sheet stretching and a critical value of shrinking velocity exists below which no real solution can be found. In "Dual approximate solutions of the unsteady viscous flow over a shrinking cylinder with optimal homotopy asymptotic method," V. Marinca and R.-D. Ene studied the unsteady viscous flow over a continuously shrinking surface with mass suction being investigated using the optimal homotopy asymptotic method (OHAM). The nonlinear differential equation is obtained by means of the similarity transformation. It was revealed that dual solutions exist for a certain range of mass suction and unsteadiness parameters and the results obtained using OHAM agreed well with the one obtained using fourth-order Runge-Kutta iteration scheme coupled with shooting method. In "Analysis of heat transfer in Berman flow of nanofluids with Navier slip, viscous dissipation, and convective cooling," $\mathrm{O}$. D. Makinde et al. investigated the combined effects of viscous dissipation, Navier slip, and convective cooling on Berman flow and heat transfer of water base nanofluids containing $\mathrm{Cu}$ and $\mathrm{Al}_{2} \mathrm{O}_{3}$ as nanoparticles. The model nonlinear differential equations are tackled both analytically using perturbation series method and numerically using Runge-Kutta-Fehlberg integration technique coupled with shooting scheme. Pertinent results with respect to effects of variation in governing parameters on the dimensionless velocity, temperature, skin friction, pressure drop, and Nusselt numbers are presented graphically and discussed quantitatively. In "Simulation of impinging cooling performance with pin fins and mist cooling adopted in a simplified gas turbine transition piece," T. Xu et al. employed a commercial computational fluid dynamics (CFD) program FLUENT to analyse the heat transfer and pressure characteristics of the impinging cooling in the coolant chamber. The simulation results reveal that the factors of detached space and pin-fin diameter ratio have a significant effect on the convective heat transfer. In "A local integral equation formulation based on moving Kriging interpolation for solving coupled nonlinear reaction-diffusion equations," K. Yimnak and A. Luadsong presented a meshless local Petrov-Galerkin method (MLPG) with the test function (in view of the Heaviside step function) to solve a system of coupled nonlinear reaction-diffusion equations in two-dimensional spaces subjected to Dirichlet and Neumann boundary conditions on a square domain. Two-field velocities are approximated by moving Kriging (MK) interpolation method for constructing nodal shape function which holds the Kronecker delta property, thereby enhancing the arrangement nodal shape construction accuracy, while the Crank-Nicolson method is chosen for temporal discretization. The nonlinear terms are treated iteratively within each time step. The numerical experiments revealed that the solutions are stable and more precise.

Finally, the papers in this special issue represent a broad spectrum of nonlinear fluid flow and heat transfer problems together with their analytical and numerical solutions. They demonstrate a wide array of new developments with respect to applications. Moreover, articles published in this special issue will contribute immensely to advancement of knowledge in the field of mathematical physics and will provide scientists, engineers, industries, research scholars, and practitioners' latest theoretical and technological achievements in fluid mechanics and their heat transfer applications.

\section{Acknowledgments}

We would like to thank the authors for their contribution and the reviewers for their collaboration.

O. D. Makinde

R. J. Moitsheki

R. N. Jana

B. H. Bradshaw-Hajek

W. A. Khan 


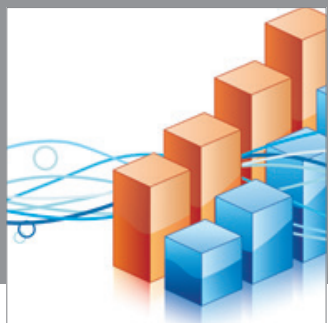

Advances in

Operations Research

mansans

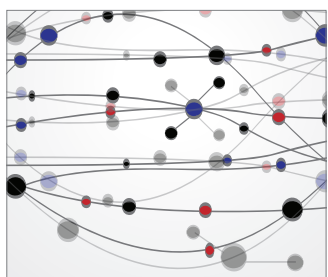

The Scientific World Journal
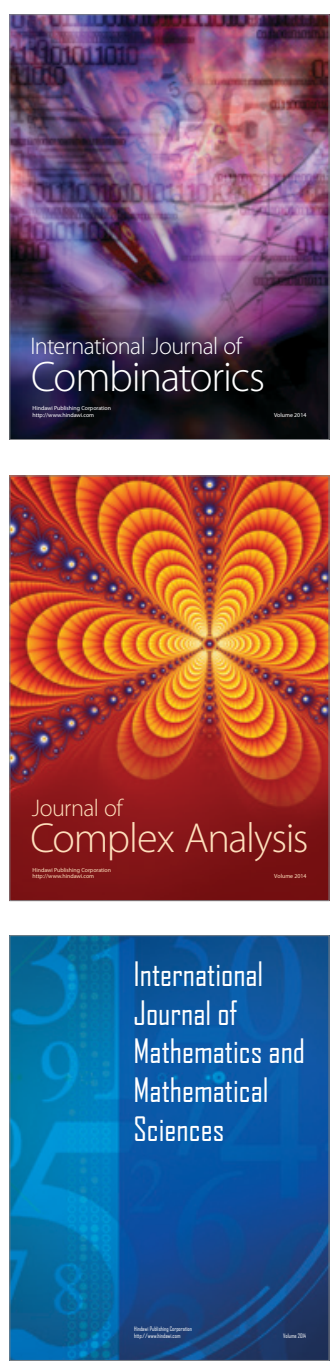
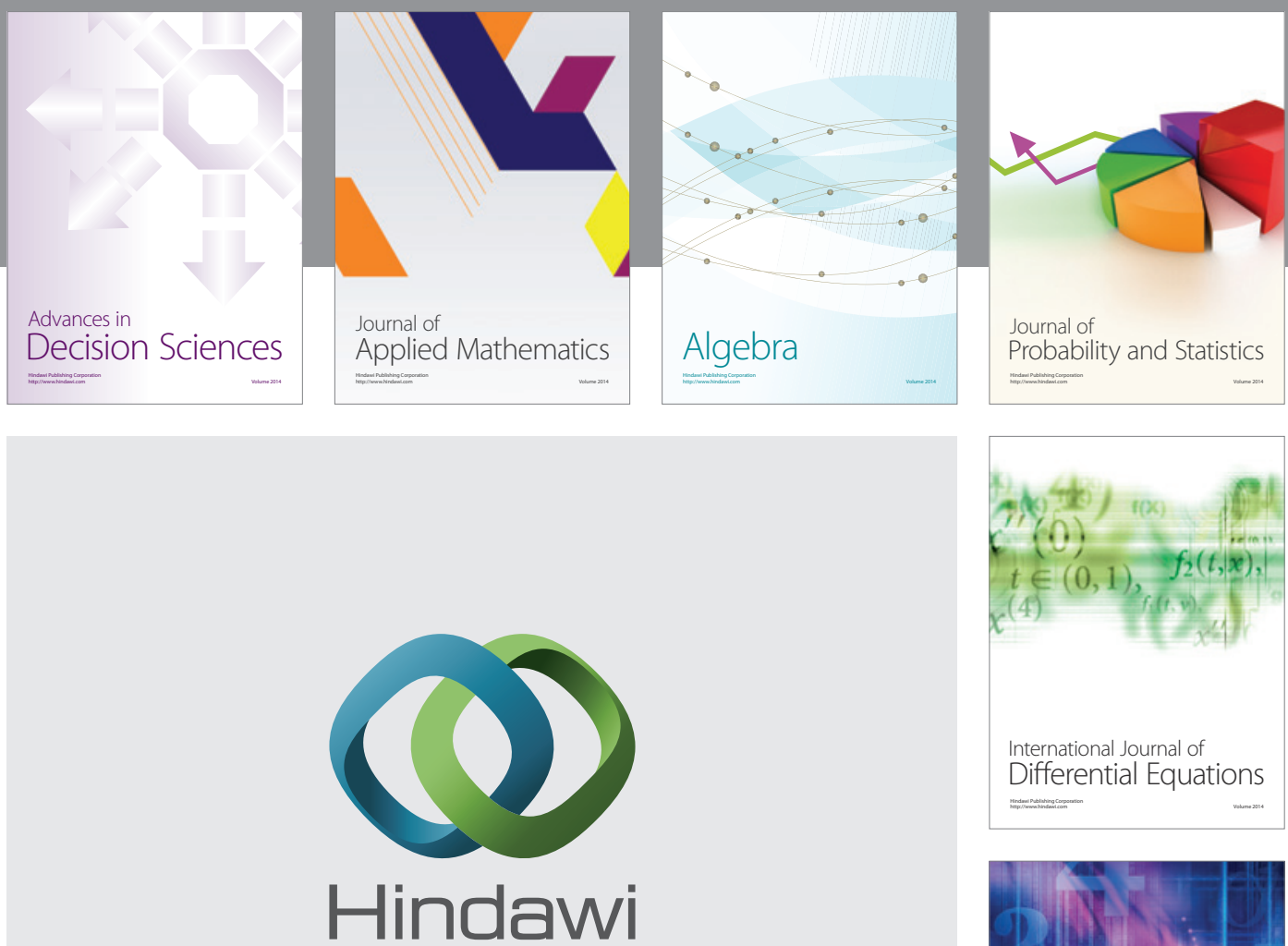

Submit your manuscripts at http://www.hindawi.com
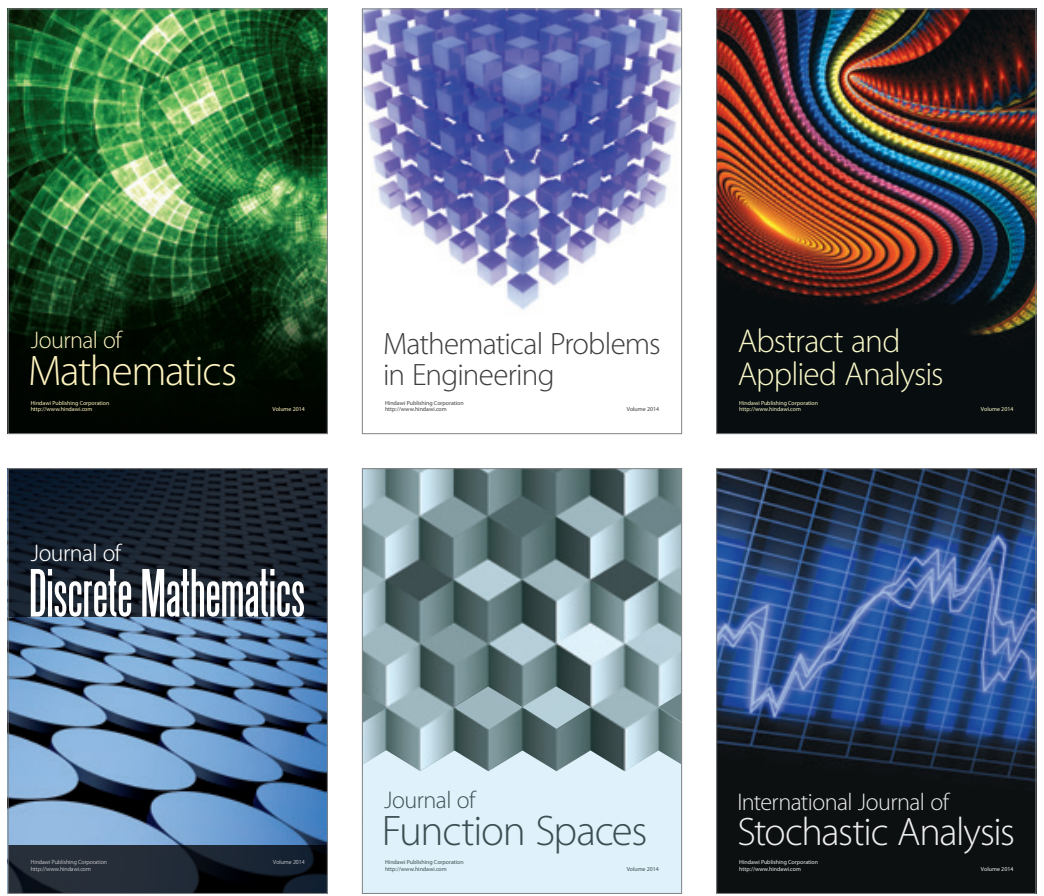

Journal of

Function Spaces

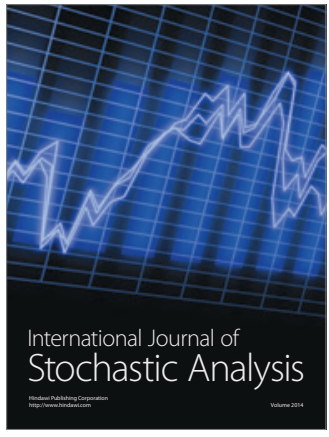

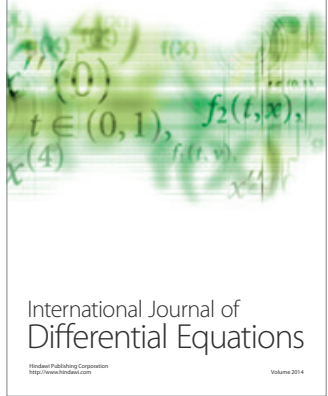
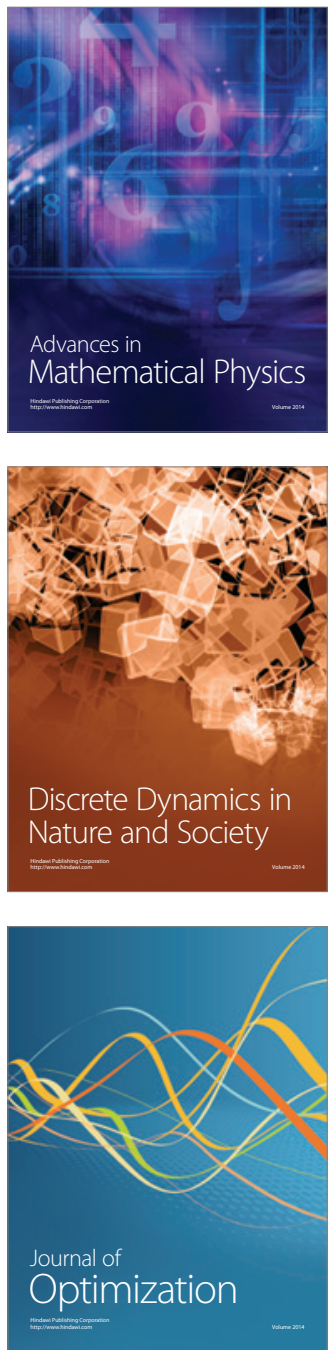